\title{
Two populations of coupled quadratic maps exhibit a plentitude of symmetric and symmetry broken dynamics
}

\author{
Cite as: Chaos 30, 033125 (2020); doi: $10.1063 / 5.0002272$ \\ Submitted: 23 January 2020 . Accepted: 28 February 2020 . \\ Published Online: 17 March 2020
}

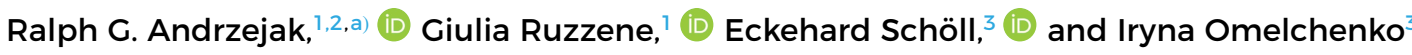

\begin{abstract}
AFFILIATIONS
${ }^{1}$ Department of Information and Communication Technologies, Universitat Pompeu Fabra, Carrer Roc Boronat 138 , 08018 Barcelona, Catalonia, Spain

${ }^{2}$ Institute for Bioengineering of Catalonia (IBEC), The Barcelona Institute of Science and Technology, Baldiri Reixac 10-12, 08028 Barcelona, Spain

${ }^{3}$ Institut für Theoretische Physik, Technische Universität Berlin, Hardenbergstrasse 36, 10623 Berlin, Germany
\end{abstract}

\begin{abstract}
a) Author to whom correspondence should be addressed: ralph.andrzejak@upf.edu
\end{abstract}
\begin{abstract}
We numerically study a network of two identical populations of identical real-valued quadratic maps. Upon variation of the coupling strengths within and across populations, the network exhibits a rich variety of distinct dynamics. The maps in individual populations can be synchronized or desynchronized. Their temporal evolution can be periodic or aperiodic. Furthermore, one can find blends of synchronized with desynchronized states and periodic with aperiodic motions. We show symmetric patterns for which both populations have the same type of dynamics as well as chimera states of a broken symmetry. The network can furthermore show multistability by settling to distinct dynamics for different realizations of random initial conditions or by switching intermittently between distinct dynamics for the same realization. We conclude that our system of two populations of a particularly simple map is the most simple system that can show this highly diverse and complex behavior, which includes but is not limited to chimera states. As an outlook to future studies, we explore the stability of two populations of quadratic maps with a complex-valued control parameter. We show that bounded and diverging dynamics are separated by fractal boundaries in the complex plane of this control parameter.
\end{abstract}

Published under license by AIP Publishing. https://doi.org/10.1063/5.0002272

Chimera states are characterized by the intriguing coexistence of synchronization and desynchronization in networks. They were first described in models with a very simple structure, namely, in ring networks of identical non-locally coupled phase oscillators, ${ }^{1,2}$ as well as in two populations of identical phase oscillators with $^{3}$ and without ${ }^{4}$ frequency mismatch between the populations. Apart from simple phase oscillators, ${ }^{3-7}$ further work on chimera states in two populations of time-continuous dynamics used phase oscillators with coupling delay, ${ }^{8,9}$ external forcing, ${ }^{9}$ or with inertia, ${ }^{10-13}$ Stuart-Landau oscillators, ${ }^{13-15}$ pulse-coupled oscillators, ${ }^{16}$ experimental mechanical ${ }^{17}$ or chemical oscillators, ${ }^{18}$ models of neurons, ${ }^{13,19,20}$ and social agents, ${ }^{21}$ among others. Extensive work is also dedicated to chimera states in networks of time-discrete maps (e.g., Refs. 22-44). There are, however, only very few studies on chimera states in two populations of maps. ${ }^{45,46}$ Here, we address this very simple setting and study two populations of real-valued quadratic maps. We show that a plentitude of distinct dynamics can be generated by varying the coupling strengths within and across the two populations. After being started with random initial conditions, the maps in individual populations can synchronize or desynchronize, and their temporal evolution can become periodic or aperiodic. Furthermore, individual populations can show combinations of synchronized and desynchronized states as well as combinations of periodic and aperiodic motions. We provide various examples for which both populations have the same type of dynamics, such as an aperiodic motion, which is synchronous within populations but asynchronous across populations. We furthermore show different chimera states, for which the symmetry between the two identical populations is broken by the network dynamics. For example, while one population can be synchronous and periodic, the other population can be asynchronous and aperiodic. A key 
point of this study is that a single and very simple system is capable of generating highly diverse and complex behavior. It is this simplicity which allows us to describe the basic mechanism behind the symmetry breaking in our system of all identical elements.

Lattices of coupled logistic maps have been shown to exhibit different scenarios of complete or partial synchronization (e.g., Refs. 47 and 48). Chimera states in a ring network of nonlocally coupled logistic maps were first described by Omelchenko and colleagues..$^{22}$ Nayak and Gupte ${ }^{45}$ reported on chimera states in two populations of sine-circle maps. The first experimental observation of chimeras in electro-optical coupled map lattices in one and two dimensions was provided by Hagerstrom et al. ${ }^{24}$ Apart from coupled logistic maps, ${ }^{22,23,27-30,37-39,41-44}$ chimera states were illustrated for coupled Henon maps, ${ }^{26,32-34,36}$ sinecircle maps, ${ }^{45,46}$ sine-squared maps, ${ }^{35,44}$ cosine maps, ${ }^{24}$ piecewise linear and logistic maps, ${ }^{25}$ and cubic maps. ${ }^{31,39,40}$ They were found for ring networks with nonlocal coupling, ${ }^{22-26,28-36,39,41-43}$ ring networks with hierarchical connectivity, ${ }^{38}$ globally coupled networks with delay, ${ }^{35}$ two-dimensional lattices, ${ }^{24,40}$ as well as two-, ${ }^{27,34,37}$ three-, ${ }^{43}$ and many-layer multiplex networks. ${ }^{39,41}$ Apart from phase chimeras, ${ }^{22-24,28-34,36,38-43}$ the existence of amplitude chimeras, ${ }^{28-32,34,36,38-41,43}$ double-well chimeras, ${ }^{31,39,40}$ nested chimera states ${ }^{38}$ splay chimeras, ${ }^{46}$ and solitary state chimeras ${ }^{34,36}$ was shown. Dynamics were found to show spatial as well as spatiotemporal chaos (e.g., Refs. 22 and 25). Further work on chimera states in maps revealed temporal intermittency, ${ }^{29,32}$ noise-induced inter-layer switching, ${ }^{44}$ and relay synchronization. ${ }^{43}$

By means of a linear change of variables, the logistic map can be transformed into the quadratic map $f(z)=z^{2}+c$, which is a particularly simple map that can show chaos. For complex-valued $c$ and $z$, the quadratic map allows one to generate the fractal Julia sets and Mandelbrot set, which made this map famous, including in popular science. ${ }^{49}$ We confine the quadratic map to real values by setting $c=-1.8$ and using real-valued initial conditions. Decreasing the control parameter from $c=0.25$ to $c=-2$ leads to a perioddoubling bifurcation scenario. For $c=-1.8$, the quadratic map shows chaotic motion, and windows of periodic solutions are closeby on the axis of the parameter $c$.

We use a network of two identical populations, $X$ and $Y$, each composed by $N=100$ identical quadratic maps. Each individual map is coupled with strength $C_{\mathrm{w}}$ to all other maps within its population and with strength $C_{\mathrm{a}}$ to all maps in the other population,

$$
\begin{aligned}
X: \quad x_{i}^{t+1}= & f\left(x_{i}^{t}\right)+\frac{C_{\mathrm{w}}}{N} \sum_{j=1}^{N}\left[f\left(x_{j}^{t}\right)-f\left(x_{i}^{t}\right)\right] \\
& +\frac{C_{\mathrm{a}}}{N} \sum_{j=1}^{N}\left[f\left(y_{j}^{t}\right)-f\left(x_{i}^{t}\right)\right], \\
Y: \quad y_{i}^{t+1}= & f\left(y_{i}^{t}\right)+\frac{C_{\mathrm{w}}}{N} \sum_{j=1}^{N}\left[f\left(y_{j}^{t}\right)-f\left(y_{i}^{t}\right)\right] \\
& +\frac{C_{\mathrm{a}}}{N} \sum_{j=1}^{N}\left[f\left(x_{j}^{t}\right)-f\left(y_{i}^{t}\right)\right] .
\end{aligned}
$$

Here, $t$ is discrete time in units of the iteration step. We use random initial conditions distributed uniformly in $[-1.5,1.5]$ for the variables $x_{i}^{t=1}$ and $y_{i}^{t=1}$. They are independent for $X$ and $Y$ and across the maps $i=1, \ldots, N$.

To characterize the network's dynamics, we first define the following time-resolved measures. The standard deviation of $x_{i}^{t}$ across nodes $(i=1, \ldots, N)$ is denoted by $\sigma_{x}^{t}$ and is used to assess the synchronization within population $X$. We get $\sigma_{x}^{t}=0$, if at time $t$ all maps are identically synchronized, regardless of whether or not their motion is periodic. For a non-synchronous motion, we get $\sigma_{x}^{t}>0$, without normalization to some upper bound. Periodicity with period $p$ is assessed by $\delta_{x, p}^{t}=\left\langle\left|x_{i}^{t}-x_{i}^{t+p}\right|\right\rangle$, where $|\cdot|$ denotes the absolute value and the angular brackets indicate averaging across nodes $(i=1, \ldots, N)$. A value of $\delta_{x, p}^{t}=0$ means that at time $t$, all maps are at the beginning of a period $p$ cycle, regardless of whether or not they are synchronized across nodes. In general, when we report the dynamics to be periodic with order $p$, we imply that $p$ is the minimum period. Temporal averages of $\sigma_{x}^{t}$ and $\delta_{x, p}^{t}$ are denoted by $\sigma_{x}$ and $\delta_{x, p}$, respectively. The quantities $\sigma_{y}^{t}, \sigma_{y}, \delta_{y, p}^{t}$, and $\delta_{y, p}$ are defined analogously for population $Y .^{50,59}$ These measures allow us to characterize different types of symmetry broken chimera states. A dynamics with broken synchronization symmetry is detected if $\sigma_{x}=0$ but $\sigma_{y}>0$, or $\sigma_{x}>0$ but $\sigma_{y}=0$. In contrast, $\sigma_{x}=\sigma_{y}=0$ or $\sigma_{x} \approx \sigma_{y}>0$ indicates that the synchronization symmetry is not broken. In an analogous way, the $\delta$ measures assess the periodicity symmetry. The $\sigma$ and $\delta$ measures are independent. For a given dynamics, the measures can, therefore, determine if both types of symmetry are broken, only one type of symmetry is broken, or if the dynamics is symmetric with regard to both synchronization and periodicity.

Before we explore the two-parameter space spanned by the coupling strengths within populations and across populations, we first show the results obtained for nine different exemplary combinations of these coupling strengths. This includes three settings in which the coupling across populations is stronger than the one within them. The respective values of $C_{\mathrm{w}}$ and $C_{\mathrm{a}}$ are given in Figs. 1-4, where we show realizations of the dynamics obtained for the nine settings. ${ }^{51}$ We use the labels 1-9 to refer to the settings in the text and to identify them in the parameter plane displayed in Fig. 5.

Figure 1(a) shows a realization of the network's dynamics obtained for setting 1 . Shortly after being started with random initial conditions, at $t \approx 20$, both populations enter into a joint asynchronous and aperiodic dynamics. As a result, we find $\sigma_{x}^{t} \approx \sigma_{y}^{t}>0$ [Fig. 1(b)] and $\delta_{x, 6}^{t} \approx \delta_{y, 6}^{t}>0$ [Fig. 1(c)]. Starting at $t \approx 50$, this initial symmetry between the two populations's dynamics is broken and both pass through some transient motions that are distinct between them. Eventually, both populations enter into a period-6 motion, $X$ at $t \approx 100$ and $Y$ at $t \approx 120$. However, while population $Y$ is synchronous across its nodes, $X$ remains asynchronous. In consequence, we get $\delta_{x, 6}^{t}=\delta_{y, 6}^{t}=0$ and $\sigma_{x}^{t}>0, \sigma_{y}^{t}=0$. This configuration remains stable, as can be seen from Fig. 1(d), which shows the same realization of the dynamics but for a later interval. Averaging across this interval, we get $\sigma_{x}=0.78$, while $\sigma_{y}, \delta_{x, 6}$, and $\delta_{y, 6}$ remain zero. Visual inspection of Fig. 1(d) might suggest that $X$ and $Y$ enter into a period- 2 and period- 3 motion, respectively. However, $\delta_{x, 2}^{t}$ and $\delta_{y, 3}^{t}$ are nonzero, and $p=6$ is the lowest period for which we obtain $\delta_{x, 6}^{t}=0$ and $\delta_{y, 6}^{t}=0$. Accordingly, for this realization, the network symmetry is broken by the dynamics 

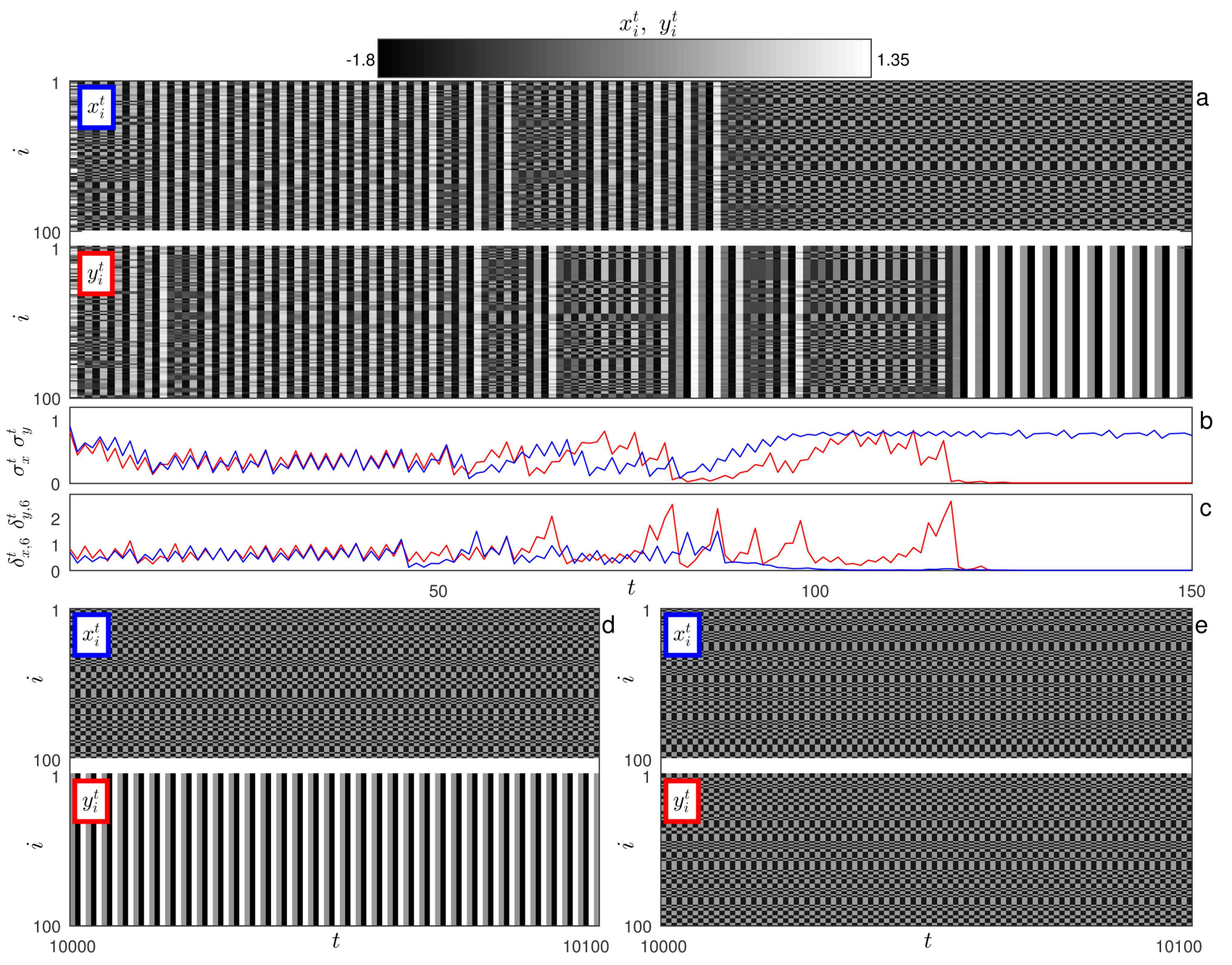

FIG. 1. Periodic dynamics with a broken synchronization symmetry coexists with globally asynchronous and periodic dynamics. Results for two exemplary realizations obtained for different random initial conditions with setting 1: $C_{w}=2.7 \times 10^{-1}$ and $C_{a}=1.2 \times 10^{-2}$. (a)-(d) First realization, (e) second realization. Panel (a)-(c) have the same abscissa. (a) Values of the maps vs discrete time $t$ and index $i$ for the populations $X$ and $Y$. (b) Synchronization measures $\sigma_{x}^{t}$ (blue) and $\sigma_{y}^{t}$ (red) for the same interval as shown in panel (a). These measures take zero values for full synchronization of the corresponding population. (c) Same as panel (b), but for the periodicity measures $\delta_{x, 6}^{t}$ (blue) and $\delta_{y, 6}^{t}$ (red), which take zero values for period-6 dynamics. (d) Same realization like in panel (a), but for a later time interval. (e) Same as panel (d), but showing the second realization of the dynamics obtained for setting 1 . We used $N=100$ and $c=-1.8$.

of its two populations with regard to their synchronization. In contrast, the symmetry is maintained with regard to the populations' periodicity. This dynamics can be regarded as analogous to phase chimeras previously described in ring networks of coupled maps. ${ }^{22-24,28-34,36,38-43}$ Figure 1 (e) shows the network dynamics still for setting 1, but after being started with a different set of random initial conditions. In contrast to the first realization discussed above [Figs. 1(a)-1(d)], for this second realization, both populations show the same type of behavior. They are periodic $\left(\delta_{x, 2}=\delta_{y, 2}=0\right)$ and asynchronous $\left(\sigma_{x}=\sigma_{y}=0.79\right)$. Hence, for this second realization, the network symmetry is not broken by the dynamics of its populations. Such a coexistence of qualitatively different dynamics for different random initial conditions is not a peculiarity of setting 1 . We found it for many of the cases we studied. This effect of initial conditions should be investigated further in future studies using methods such as those used in Refs. 52-54. In the following examples, we restrict ourselves to one realization for each setting, each selected to illustrate a different type of dynamics.

Figure 2(a) displays a realization for setting 2. During approximately the first 80 iterations, both populations behave qualitatively similar. An increasing number of nodes engages to a motion, which is almost periodic in time with $p=6$ and synchronous across nodes. 

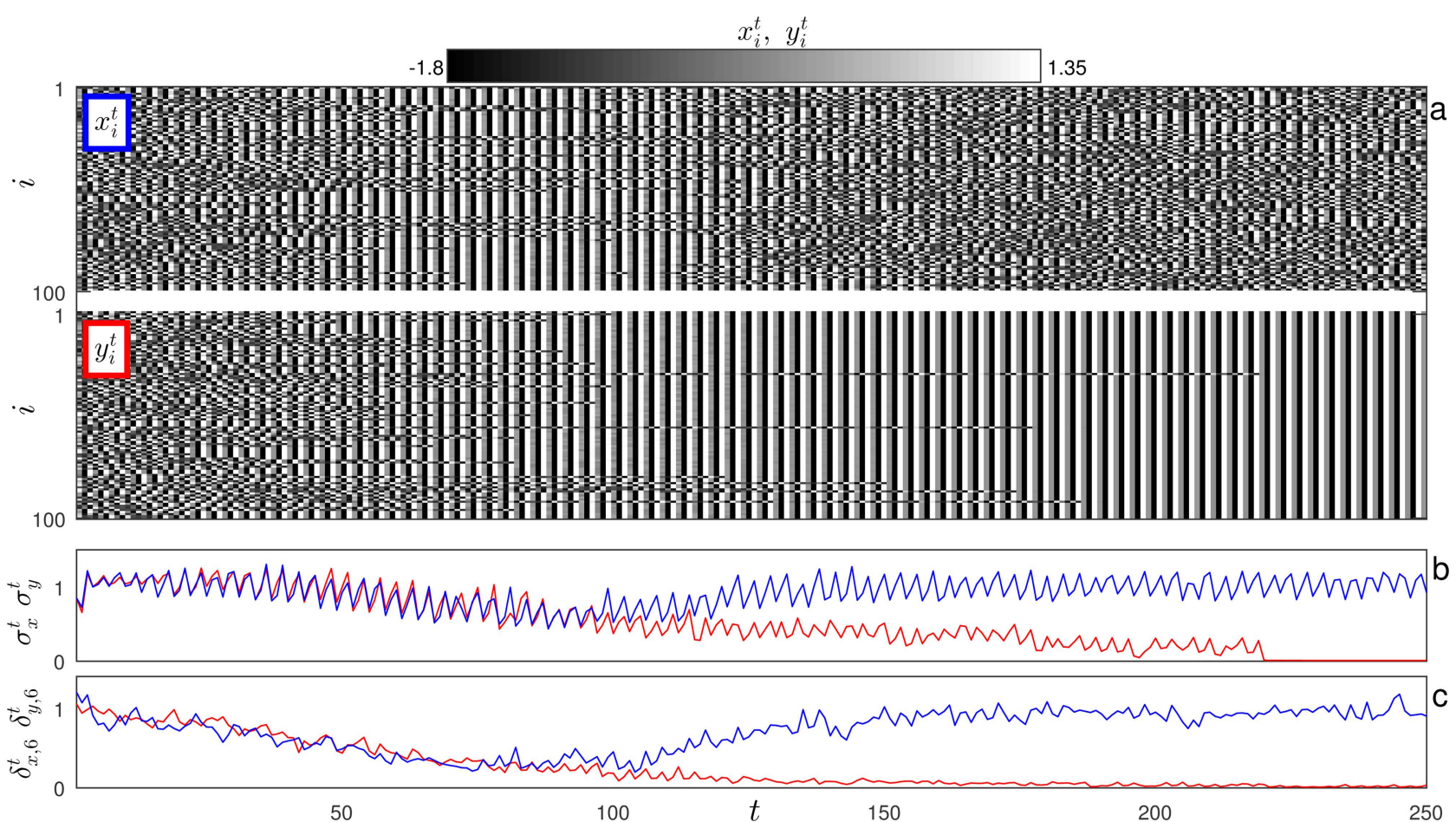

FIG. 2. Dynamics with broken synchronization symmetry and broken periodicity symmetry. Panels (a)-(c) are analogous to Figs. 1 (a) -1 (c), but here for an exemplary realization of setting 2: $C_{w}=1.7 \times 10^{-4}$ and $C_{a}=1.7 \times 10^{-2}$ with $N=100$ and $c=-1.8$.

As a result, $\sigma_{x}^{t}, \sigma_{y}^{t}, \delta_{x, 6}^{t}$, and $\delta_{y, 6}^{t}$ all decrease during this initial phase [Figs. 2(b) and 2(c)]. Furthermore, this emerging pattern is locked across the two populations. Subsequently, in population $Y$, more and more nodes join, and eventually all nodes are synchronized. For $t>220$, we, therefore, get $\sigma_{y}^{t}=0$ and $\delta_{y, 6}^{t} \approx 0.01$. The contrary behavior is observed for population $X$. From $t \approx 80$ to $t \approx 150$, all nodes return to an asynchronous and aperiodic motion. The values of $\sigma_{x}^{t}$ and $\delta_{x, 6}^{t}$ approach the ones they had for random initial conditions at $t=1$. After these initial transients, population $X$ evolves as asynchronous and aperiodic, while $Y$ is synchronous and almost periodic. Population $Y$ cannot settle to a fully periodic dynamics due to the aperiodic input from population $X$, but the deviations from a period- 6 motion remain very small, $\delta_{y, 6}=0.01$. Accordingly, in this example, the symmetry is broken with regard to both the synchronization and periodicity of the two populations. It is analogous to amplitude chimeras in ring networks of coupled maps. ${ }^{28-32,34,36,38-41,43}$

Above, we already included a dynamics in which the two populations behave qualitatively the same, namely, periodic and asynchronous [second realization of setting 1, see again Fig. 1(e)]. Further symmetric dynamics are shown in Fig. 3. Setting 3 allows generating aperiodic and asynchronous dynamics [Fig. 3(a)]. Aperiodic dynamics, which are synchronous within populations but asynchronous across the populations, can be obtained with setting 4 [Fig. 3(b)]. Setting 5 results in aperiodic and globally synchronous dynamics [Fig. 3(c)]. A realization obtained for setting 6 is shown in Fig. 3(d). Both populations are synchronous and periodic with $p=3$. While at first sight, there seems to be a lag-1 synchronization between $X$ and $Y$, the map values are, in fact, different for the two populations. This is, however, difficult to perceive at the resolution of the gray scale for their display. Setting 7 yields an example for a blended pattern resulting in an intermediate degree of periodicity and synchronization [Fig. 3(e)]. This pattern resembles the initial transient of setting 2 (see again Fig. 2), but remains stable for this realization of setting 7 . At first sight, the realization obtained for setting 8 [Fig. 3(f)] looks like a periodic and globally synchronous dynamics. Indeed, all maps are in a period-6 motion. However, two maps in $X$ and three maps in $Y$ are detached from their respective population and are synchronized to each other instead. Accordingly, this dynamics represents solitary states (cf. Refs. 34, 36, and 55). Furthermore, a closer inspection of the values reveals that neither the motion of the detached maps nor the motion of the remaining majority of maps is synchronized across $X$ and $Y$. As last example, we show a realization of the dynamics obtained for setting 9 [Fig. 4]. In contrast to the examples we described above, this dynamics does not settle to a stable configuration. After initial transients, population $X$ synchronizes at $t \approx 750$, while $Y$ remains asynchronous $\left(\sigma_{x}^{t}=0\right.$, $\left.\sigma_{y}^{t}>0\right)$. Furthermore, $Y$ remains aperiodic $\left(\delta_{y, 12}^{t}>0\right)$. In contrast, population $X$ switches intermittently between an aperiodic motion (e.g., $\delta_{x, 3}^{t}>0$ between $1480<t<1623$ ) and an almost periodic motion (e.g., $\delta_{x, 3}^{t} \approx 0$ between $1624<t<2513$ ). Such intermittent behavior was described for chimeras in ring networks of maps ${ }^{29,32}$ and two populations of phase oscillators with inertia ${ }^{11,12}$ before. 


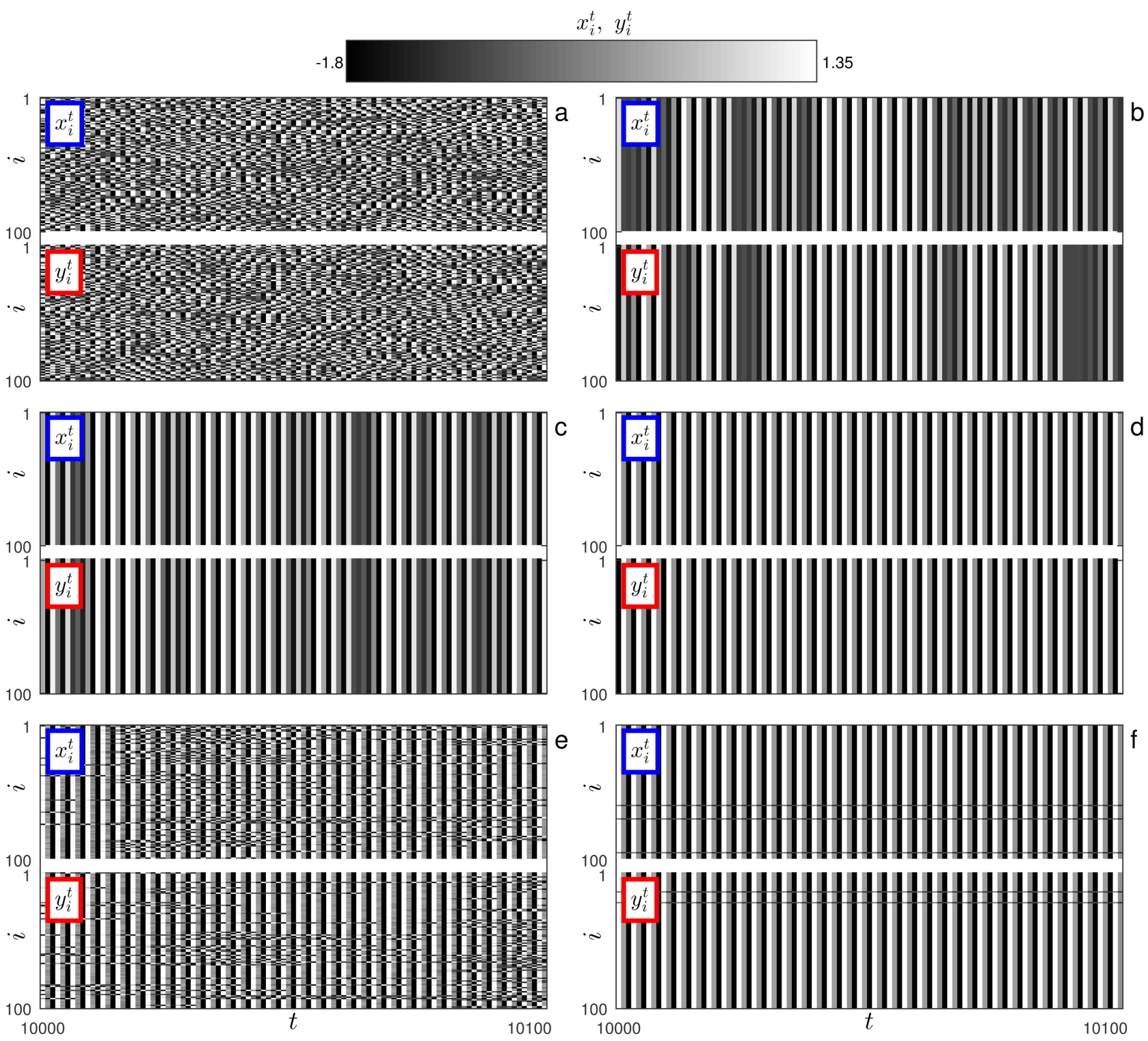

FIG. 3. A variety of dynamics without symmetry breaking. All panels display values of the maps vs discrete time $t$ and index $i$ for the populations $X$ and $Y$. Panel (a): Setting 3. $C_{w}=3.8 \times 10^{-4}$ and $C_{a}=9.5 \times 10^{-5}$. We get $\sigma_{x}=\sigma_{y}=1.1, \delta_{x, 12}=1.0$, and $\delta_{y, 12}=1.1$. For such aperiodic dynamics, we report $\delta_{x, 12}$ and $\delta_{x, 12}$, since this includes $p \in\{2,3,4,6,12\}$. Panel (b): Setting 4. $C_{w}=4.7 \times 10^{-1}, C_{a}=5.9 \times 10^{-5}, \sigma_{x}=\sigma_{y}=0, \delta_{x, 12}=1.1$, and $\delta_{y, 12}=0.69$. Panel (c): Setting 5. $C_{w}=2.0 \times 10^{-1}$, $C_{a}=2.5 \times 10^{-1}, \sigma_{x}=\sigma_{y}=0$, and $\delta_{x, 12}=\delta_{y, 12}=1.3$. Panel (d): Setting 6. $C_{w}=4.9 \times 10^{-1}, C_{a}=7.8 \times 10^{-3}, \sigma_{x}=\sigma_{y}=0$, and $\delta_{x, 3}=\delta_{y, 3}=0$. Panel (e): Setting 7. $C_{\mathrm{w}}=1.3 \times 10^{-2}, C_{a}=6.3 \times 10^{-3}, \sigma_{x}=0.70, \sigma_{y}=0.66, \delta_{x, 6}=0.33$, and $\delta_{y, 6}=0.32$. Panel (f): Setting 8. $C_{w}=3.2 \times 10^{-1}, C_{a}=2.9 \times 10^{-5}, \sigma_{x}=0.22, \sigma_{y}=0.18$, and $\delta_{x, 3}=\delta_{y, 3}=0$. In all panels: $N=100$ and $c=-1.8$.

In order to describe the mechanism behind our results, we write the network equations [Eqs. (1) and (2)] in a different form (cf. Refs. 23, 28, 30, 33, 38, 40, 41, 43, and 45). Defining $\gamma=1-C_{\mathrm{w}}-$ $C_{\mathrm{a}}, F\left(X^{t}\right)=\frac{1}{N} \sum_{j=1}^{N} f\left(x_{j}^{t}\right)$, and $F\left(Y^{t}\right)=\frac{1}{N} \sum_{j=1}^{N} f\left(y_{j}^{t}\right)$, we get

$$
X: \quad x_{i}^{t+1}=\gamma f\left(x_{i}^{t}\right)+C_{\mathrm{w}} F\left(X^{t}\right)+C_{\mathrm{a}} F\left(Y^{t}\right),
$$

$$
Y: \quad y_{i}^{t+1}=\gamma f\left(y_{i}^{t}\right)+C_{\mathrm{w}} F\left(Y^{t}\right)+C_{\mathrm{a}} F\left(X^{t}\right),
$$

where $f(z)=z^{2}+c$ continues to be the quadratic map. When regarded in isolation, and for the ranges of $C_{\mathrm{w}}$ and $C_{\mathrm{a}}$ used here, the rescaled map $\gamma f$ exhibits a period-doubling bifurcation scenario very similar to the one of $f$. However, the fine structure of the bifurcation diagram and the positions of the bifurcation points with 


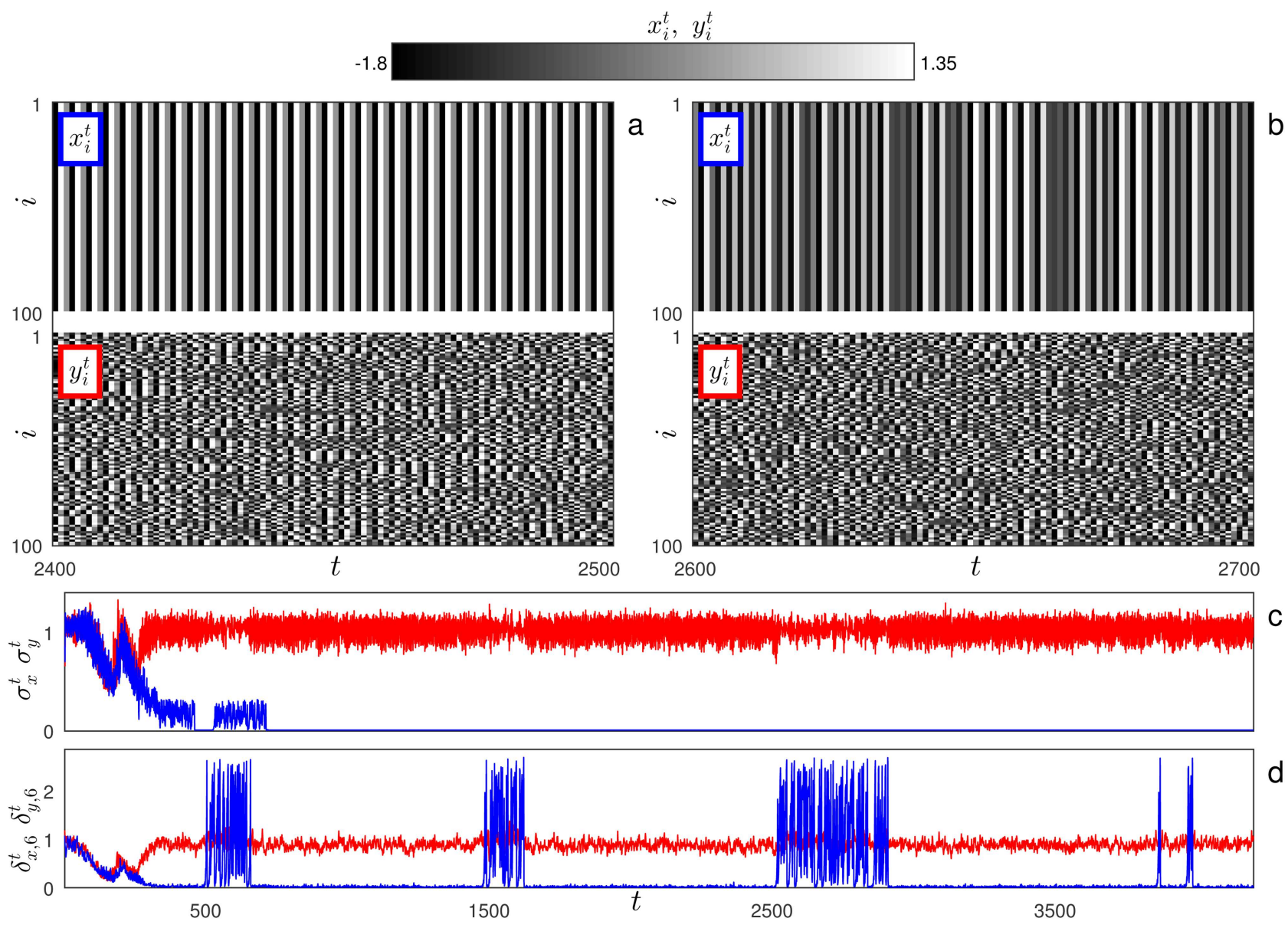

FIG. 4. Intermittent switching between different dynamics. Panels (a) and (b) are analogous to the graphics in Fig. 3, but here for two intervals of one realization of setting 9 $\left(C_{w}=3.7 \times 10^{-5}, C_{a}=1.9 \times 10^{-2}, N=100\right.$, and $\left.c=-1.8\right)$. (c) Synchronization measures $\sigma_{x}^{t}$ (blue) and $\sigma_{y}^{t}$ (red) for a longer interval, including the intervals of panels (a) and (b). (d) Same as panel (c), but for the periodicity measures $\delta_{x, 6}^{t}$ (blue) and $\delta_{y, 6}^{t}$ (red).

regard to the parameter $c$ depend on $\gamma$ (results not shown). This already leads to an impact of the coupling strengths $C_{\mathrm{w}}$ and $C_{\mathrm{a}}$ on the characteristics of the dynamics. What is more important is the input by $F\left(X^{t}\right)$ and $F\left(Y^{t}\right)$. At the level of individual maps, they add to the period-doubling bifurcation parameter $c$. Therefore, even smallest differences in these terms can push the map from a periodic to a chaotic regime or vice versa. The fact that these terms are time-dependent increases the complexity further. At the level of the network dynamics, the $F\left(X^{t}\right)$ and $F\left(Y^{t}\right)$ terms break the symmetry between the two populations since their positions are crossed over in Eqs. (3) and (4). Therefore, once $X$ and $Y$ enter into different types of dynamics, also $F\left(X^{t}\right)$ and $F\left(Y^{t}\right)$ differ, resulting in a different input for $X$ and $Y$. Like reasoned above, these different inputs can potentially push $X$ and $Y$ into different dynamical regimes, again leading to different $F\left(X^{t}\right)$ and $F\left(Y^{t}\right)$, and so on. This process may then either amplify or reduce the differences in the dynamics of $X$ and $Y$. For certain values of $C_{\mathrm{w}}$ and $C_{\mathrm{a}}$ in combination with some initial conditions, this mechanism leads to the stabilization of a symmetry broken dynamics [see again first realization of setting 1 in Figs. $1(\mathrm{a})-1(\mathrm{~d})$ ]. For the same $C_{\mathrm{w}}$ and $C_{\mathrm{a}}$, but in combination with other transitory dynamics, the difference in $X$ and $Y$ can fade out. Accordingly, an initial symmetry breaking between $X$ and $Y$ does not always lead to the stabilization of a symmetry broken chimera state. Instead, the system can likewise settle to a symmetric dynamics [see again second realization of setting 1 in Fig. 1(e)]. For other $C_{\mathrm{w}}$ and $C_{\mathrm{a}}$, only symmetry broken (see again setting 2 in Fig. 2 ) or only symmetric patterns are stable [see again settings 3-7 in Figs. 3(a)-3(e). Setting 8 shows a plentitude of qualitatively different patterns for different initial conditions, only one of which is displayed in Fig. 3(f)]. Finally, again for other combinations of the coupling strengths, the dynamics might not settle down to a stable pattern but switch intermittently between different dynamical regimes (see again setting 9 in Fig. 4).

After inspecting details of individual realizations for exemplary pairings of $C_{\mathrm{w}}$ and $C_{\mathrm{a}}$, we now explore the full two-parameter plane spanned by these coupling strengths. Figure 5(a) allows identifying 

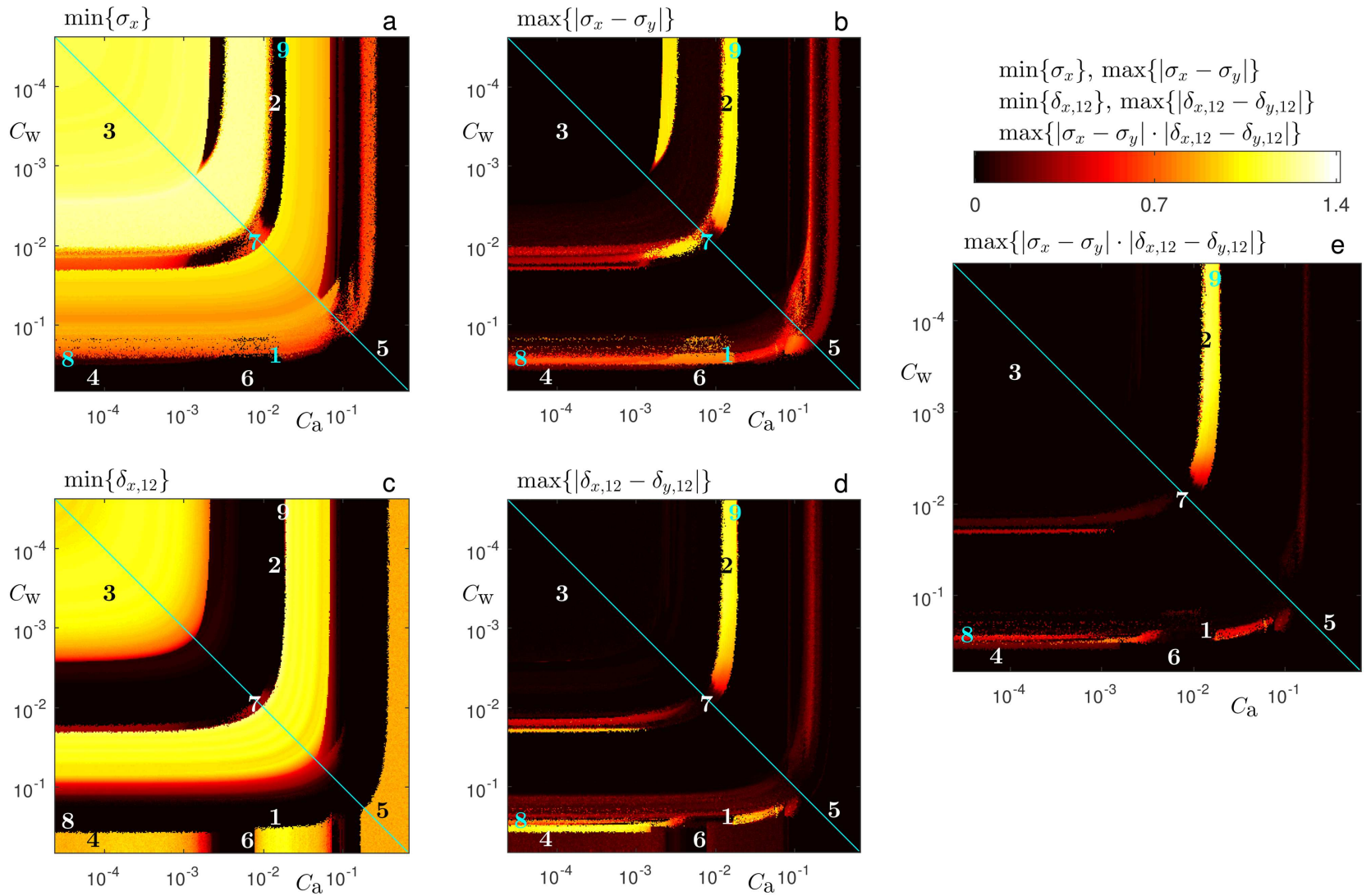

FIG. 5. Synchronization (a) and periodicity (c) of individual populations as well as the symmetry breaking of these properties across populations [(b), (d), and (e)] in dependence on the coupling strengths. We vary the coupling within populations $C_{w}$ and across populations $C_{a}$ equidistantly spaced in 366 steps on a logarithmic scale but keep $N=100$ and $c=-1.8$. The labels 1-9 mark the combinations of $C_{w}$ and $C_{a}$ used for the nine settings discussed in the text and used as examples in Figs. 1-4. They are in the same positions in all panels, and their different colors are only used to enhance readability. The cyan diagonal marks $C_{w}=C_{a}$. For each combination, we generate 50 independent realizations of the network dynamics [Eqs. (1) and (2)] for 50 different random initial conditions, where the same 50 realizations are used across all panels. For each realization, we then compute the temporal averages $\sigma_{x}, \sigma_{y}, \delta_{x, p}$, and $\delta_{y, p}$ for $t \in[10000,12000]$. In all panels, the following maximal and minimal values are taken across the 50 realizations. (a) Zero values of $\min \left\{\sigma_{x}\right\}$ indicate that $X$ exhibits a fully synchronous motion for at least one of the realizations. (b) At the nonzero $\max \left\{\left|\sigma_{x}-\sigma_{y}\right|\right\}$ values, the symmetry between the populations is broken with regard to their synchronization for at least one realization. If, for a given combination of $C_{w}$ and $C_{a}$, a certain realization results in the minimal value in panel (a), this does neither imply nor rule out that the same realization leads to the maximum value in panel (b). The reason is that panel (b) does not only depend on $\sigma_{x}$ but also on $\sigma_{y}$. The panels (c) and (d) are analogous to (a) and (b), but based on the measures of periodicity $\delta_{x, 12}$ and $\delta_{y, 12}$. (e) Nonzero $\max \left\{\left|\sigma_{x}-\sigma_{y}\right| \cdot\left|\delta_{x, 12}-\delta_{y, 12}\right|\right\}$ values indicate that the symmetry was broken for both synchronization and periodicity in at least one realization. If, for a given combination of $C_{w}$ and $C_{a}$, a certain realization results in the maximum value in panel (b), this does neither imply nor rule out that the same realization leads to the maximum value in panel (e). Both $\left|\sigma_{x}-\sigma_{y}\right|$ and $\left|\delta_{x, 12}-\delta_{y, 12}\right|$, as opposed to only one of these differences, have to be high to reach the maxima displayed in panel (e). Analogous relations hold for panels (d) and (e).

the regions in this parameter plane for which $X$ shows a fully synchronous motion for at least one of 50 independent realizations (dark brown). These are complemented by regions for which we obtain only dynamics with various degrees of desynchronization (red, yellow to white). The strongest degrees of synchronization symmetry breaking between the populations [yellow regions in Fig. 5(b)] are found for a subset of parameter settings at which individual populations can show fully synchronous dynamics [dark brown regions in Fig. 5(a)]. A certain symmetry across the diagonals in the results in Figs. 5(a) and 5(b) can be attributed to the influence of $\gamma$, which is symmetric in $C_{\mathrm{w}}$ and $C_{\mathrm{a}}$. In contrast, the driving caused by $F\left(X^{t}\right)$ and $F\left(Y^{t}\right)$ in Eqs. (3) and (4) is not symmetric in $C_{\mathrm{w}}$ and $C_{\mathrm{a}}$. Somewhat more complicated patterns are seen above the diagonals in Figs. 5(a) and 5(b), i.e., where the coupling across populations is stronger than the one within populations. The panels Figs. 5(c) and 5(d) are analogous to Figs. 5(a) and 5(b), but based on the measures of periodicity. We observed dynamics with periods $p \in 2,3,4,6,12$, and here depict results for $p=12$, because this includes all lower order periods. With regard to periodicity, more complex patterns, including two periodic but not symmetry broken windows centered around $C_{\mathrm{w}} \approx 4.7 \times 10^{-1}, C_{\mathrm{a}} \approx 4.7 \times 10^{-3}$, and $C_{\mathrm{w}} \approx 3.6 \times 10^{-1}, C_{\mathrm{a}} \approx 1.2 \times 10^{-1}$ are seen below the diagonals. 

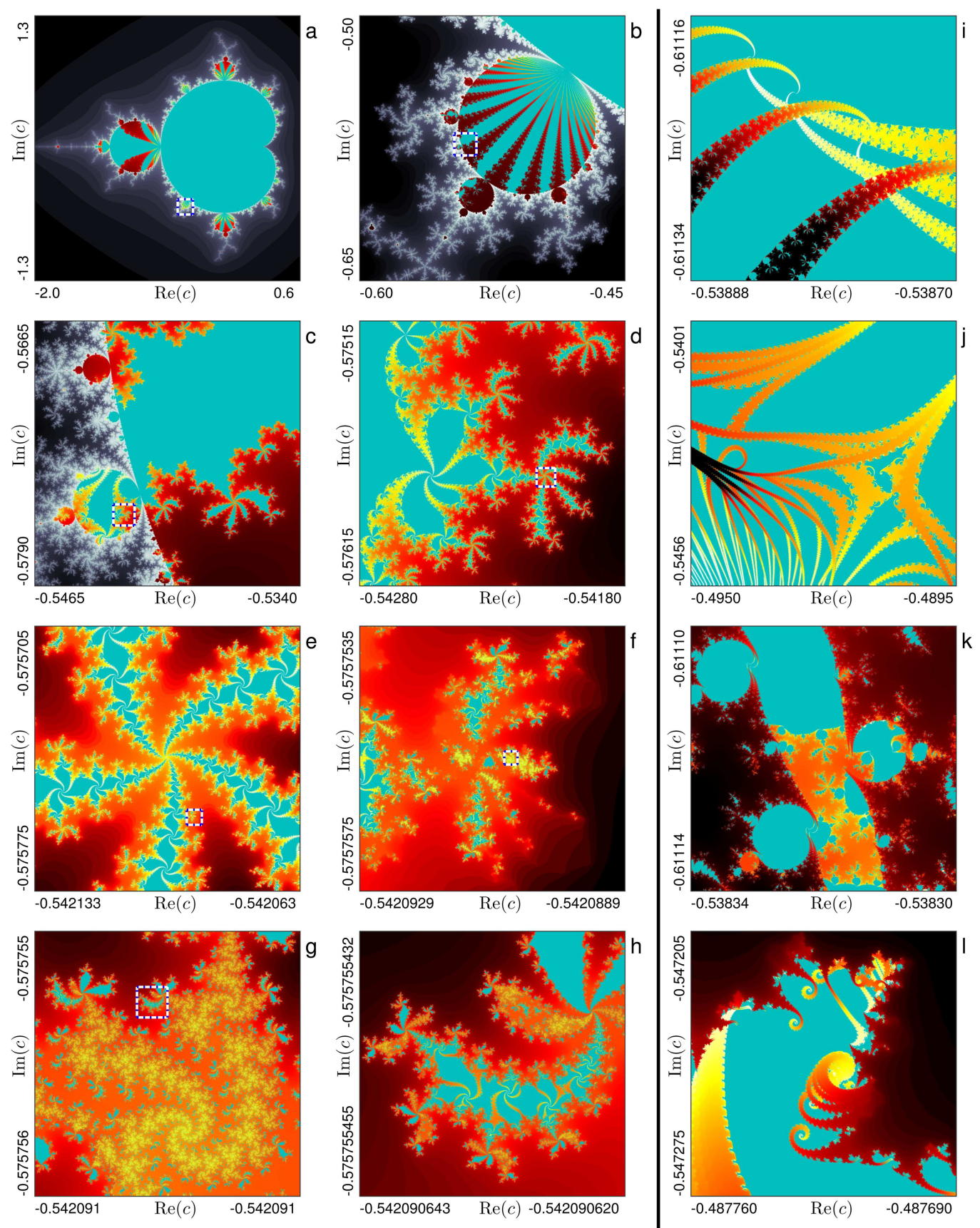

FIG. 6. Octopus's garden. In panels (a)-(c), gray colors show the complement of the Mandelbrot set, i.e., the set of complex $c$ for which the values of an individual quadratic map initiated at $z=0$ diverge to infinity. Darker gray indicates a faster divergence. Turquoise areas inside the Mandelbrot set show the set of complex $c$ for which the iterates of the two populations of coupled maps [Eqs. (1) and (2)] remain bounded for all times. Dark brown to yellow colors are used for those $c$ for which these iterates diverge to infinity, where yellow colors indicate a slower divergence. Panels $(a)-(h)$ are obtained for $C_{w}=2.7 \times 10^{-1}$ and $C_{a}=1.2 \times 10^{-2}$ (setting 1). Panels (b)-(h) are successive zooms, magnifying the dashed squares in the preceding panels (a)-(g). Axes labels indicate $c$ values in the outermost corners. Panels (i)-(l) are obtained for $C_{w}=1.7 \times 10^{-4}$ and $C_{a}=1.7 \times 10^{-2}$ (setting 2). Here, the instabilities can collide, cross, and overlay each other. In each panel, we use different color codes to enhance the contrast. Initial conditions are always generated in the following way. We start the uncoupled quadratic map with $z=0$ and take the $i$-th and $i-1$ th iterate as initial condition for the ith map in $X$ and $Y$, respectively $(i=1, \ldots, N=100)$. To allow to better resolve the details of these patterns, all panels are magnified in Figs. 1-12 in the supplementary material. 
The most prominent region of periodicity symmetry breaking, on the other hand, is found above the diagonal and coincides with a region of synchronization symmetry breaking (the region including settings 2 and 9 and limited from below by setting 7). That does not necessarily mean that the symmetry was broken in both properties. Likewise, the symmetry could be broken for the synchronization in some realizations and for the periodicity in some other realizations. However, as revealed by panel Fig. 5(e), this region indeed comprises realizations of the dynamics for which the symmetry is broken for both synchronization and periodicity. At the diagonals of the plots, we have $C_{\mathrm{w}}=C_{\mathrm{a}}$, corresponding to a network with one population of identical and identically coupled maps. The maximal across-population differences of the synchronization and periodicity measures [Figs. 5(b), 5(d), and 5(e)] can nonetheless be non-zero since the values for individual populations are non-zero and show finite-size fluctuations across the two populations. As a whole, the results shown in Fig. 5, complement the findings first described by Omelchenko et al. ${ }^{22,23}$ and Hagerstrom et al. ${ }^{24}$ according to which chimera states can be found at the transition between spatial coherence and incoherence in rings and lattices of coupled maps. We here find chimera states at the transition between synchronous vs asynchronous and periodic vs aperiodic motions.

In summary, we show that two populations of coupled quadratic maps show a plentitude of symmetric dynamics and symmetry broken chimera states. These patterns arise spontaneously from random initial conditions, i.e., there is no need to craft initial conditions to induce them. Abrams et al. ${ }^{4}$ argued that their system of two populations of coupled phase oscillators was the simplest system that supports chimera states. Since our system is based on a time-discrete map, and moreover on a particularly simple map, we can claim that our system is even simpler than the one of Abrams and colleagues. In fact, the results which we present here are supposedly only the tip of the iceberg of the system's complexity. First of all, we only show selected realizations for nine different combinations of the coupling strengths within and across population. We observed further types of symmetric and asymmetric patterns, which are not shown here for the sake of conciseness. We assume that yet unobserved patterns can be found upon further exploration of the parameter space spanned not only by the coupling strengths $C_{\mathrm{w}}$ and $C_{\mathrm{a}}$ but also by the system size $N$ and control parameter of individual maps $c$. This might also include dynamics, which we could not find despite actively searching for them, such as dynamics for which the symmetry is broken with regard to their periodicity but not with regard to their synchronization. Furthermore, we only studied a limited number of iterations. The dynamics we illustrate here are typically stabilized after some hundreds of iterations. However, we cannot rule out that some of these dynamics are, in fact, long transients that eventually collapse to some other dynamics (cf. Refs. 11, 12, 29, 32, and 56).

Finally, so far, we have only used a real-valued $c$ for the quadratic map $f(z)=z^{2}+c$. It remains to be explored what types of dynamics can be discovered for complex values of $c$. The Mandelbrot set is defined by the set of complex $c$ for which the values of the quadratic map initiated at $z=0$ remain bounded for all times. For those $c$ that form the complement of the Mandelbrot set, the map values diverge to infinity. ${ }^{49}$ As an outlook, we present an analysis (Fig. 6) of the complex values of $c$ from within the Mandelbrot set.
We determine for which of these $c$ the values of our two populations of coupled quadratic maps remain bounded. This approach is different from ring networks of $N$ locally coupled quadratic maps for which the complex control parameters are varied independently for each map yielding a spatial Mandelbrot set of dimensionality $2 N .^{57,58}$ Since we use the same control parameter for all maps, we can display the results in the complex plane. We find that the $c$ values for which the dynamics remain bounded and those for which the dynamics escapes to infinity are separated by fractal boundaries fragmenting the Mandelbrot set in a rich variety of ways (Fig. 6). Evidently, our system inherits this capacity to generate a fractal structure from the quadratic map. The high dimensionality of our system, however, leads to additional complexity, and a study of these intriguing fractal, self-similar patterns, which at some scales resemble octopus tentacles, remains the subject for future work.

\section{SUPPLEMENTARY MATERIAL}

See the supplementary material consisting of Figs. 1-12 for enlarged displays of the 12 panels of Fig. 6.

\section{ACKNOWLEDGMENTS}

We acknowledge funding from the Spanish Ministry of Economy and Competitiveness under Grant No. FIS2014-54177-R (R.G.A. and G.R.), the CERCA Programme of the Generalitat de Catalunya (R.G.), and the Deutsche Forschungsgemeinschaft (DFG) under Project No. 163436311-SFB 910 (E.S. and I.O.). We are grateful to Christian Rummel for useful discussions on the manuscript.

\section{REFERENCES}

${ }^{1}$ Y. Kuramoto and D. Battogtokh, "Coexistence of coherence and incoherence in nonlocally coupled phase oscillators," Nonlinear Phenom. Complex Syst. 5, 380-385 (2002).

${ }^{2}$ D. M. Abrams and S. H. Strogatz, "Chimera states for coupled oscillators," Phys. Rev. Lett. 93, 174102 (2004).

${ }^{3}$ E. Montbrió, J. Kurths, and B. Blasius, "Synchronization of two interacting populations of oscillators,” Phys. Rev. E 70, 056125 (2004).

${ }^{4}$ D. Abrams, R. Mirollo, S. Strogatz, and D. Wiley, "Solvable model for chimera states of coupled oscillators," Phys. Rev. Lett. 101, 084103 (2008).

${ }^{5}$ A. Buscarino, M. Frasca, L. V. Gambuzza, and P. Hövel, "Chimera states in timevarying complex networks,” Phys. Rev. E 91, 022817 (2015).

${ }^{6}$ E. A. Martens, C. Bick, and M. J. Panaggio, "Chimera states in two populations with heterogeneous phase-lag,” Chaos 26, 094819 (2016).

${ }^{7}$ M. J. Panaggio, D. M. Abrams, P. Ashwin, and C. R. Laing, "Chimera states in networks of phase oscillators: The case of two small populations," Phys. Rev. E 93, 012218 (2016).

${ }^{8}$ J. H. Sheeba, V. K. Chandrasekar, and M. Lakshmanan, "Globally clustered chimera states in delay-coupled populations,” Phys. Rev. E 79, 055203 (2009).

${ }^{9} \mathrm{R}$. Ma, J. Wang, and Z. Liu, "Robust features of chimera states and the implementation of alternating chimera states," Europhys. Lett. 91, 40006 (2010).

${ }^{10}$ T. Bountis, V. G. Kanas, J. Hizanidis, and A. Bezerianos, "Chimera states in a two-population network of coupled pendulum-like elements," Eur. Phys. J. Spec. Top. 223, 721 (2014).

${ }^{11}$ S. Olmi, "Chimera states in coupled Kuramoto oscillators with inertia," Chaos 25, 123125 (2015).

${ }^{12}$ S. Olmi, E. A. Martens, S. Thutupalli, and A. Torcini, "Intermittent chaotic chimeras for coupled rotators," Phys. Rev. E 92, 030901 (2015).

${ }^{13}$ C. R. Laing, "Dynamics and stability of chimera states in two coupled populations of oscillators," Phys. Rev. E 100, 042211 (2019). 
${ }^{14}$ C. R. Laing, "Chimeras in networks of planar oscillators," Phys. Rev. E 81, 066221 (2010).

${ }^{15}$ K. Premalatha, V. Chandrasekar, M. Senthilvelan, and M. Lakshmanan, "Chimeralike states in two distinct groups of identical populations of coupled Stuart-Landau oscillators," Phys. Rev. E 95, 022208 (2017).

${ }^{16} \mathrm{D}$. Pazó and E. Montbrió, "Low-dimensional dynamics of populations of pulsecoupled oscillators," Phys. Rev. X 4, 011009 (2014).

${ }^{17}$ E. A. Martens, S. Thutupalli, A. Fourrière, and O. Hallatschek, "Chimera states in mechanical oscillator networks," Proc. Natl. Acad. Sci. U.S.A. 110, 10563-10567 (2013).

${ }^{18} \mathrm{M}$. R. Tinsley, S. Nkomo, and K. Showalter, "Chimera and phase-cluster states in populations of coupled chemical oscillators," Nat. Phys. 8, 662-665 (2012).

${ }^{19} \mathrm{~S}$. Olmi, A. Politi, and A. Torcini, "Collective chaos in pulse-coupled neural networks," Europhys. Lett. 92, 60007 (2010).

${ }^{20}$ T. A. Glaze, S. Lewis, and S. Bahar, "Chimera states in a Hodgkin-Huxley model of thermally sensitive neurons," Chaos 26, 083119 (2016).

${ }^{21}$ J. C. González-Avella, M. G. Cosenza, and M. S. Miguel, "Localized coherence in two interacting populations of social agents," Physica A 399, 24 (2014).

${ }^{22}$ I. Omelchenko, Y. Maistrenko, P. Hövel, and E. Schöll, "Loss of coherence in dynamical networks: Spatial chaos and chimera states," Phys. Rev. Lett. 106, 234102 (2011)

${ }^{23}$ I. Omelchenko, B. Riemenschneider, P. Hövel, Y. Maistrenko, and E. Schöll, "Transition from spatial coherence to incoherence in coupled chaotic systems," Phys. Rev. E 85, 026212 (2012).

${ }^{24}$ A. M. Hagerstrom, T. E. Murphy, R. Roy, P. Hövel, I. Omelchenko, and E. Schöll, "Experimental observation of chimeras in coupled-map lattices," Nat. Phys. 8, 658-661 (2012).

${ }^{25}$ D. Dudkowski, Y. Maistrenko, and T. Kapitaniak, "Different types of chimera states: An interplay between spatial and dynamical chaos," Phys. Rev. E 90, 032920 (2014).

${ }^{26} \mathrm{~N}$. Semenova, A. Zakharova, E. Schöll, and V. Anishchenko, "Does hyperbolicity impede emergence of chimera states in networks of nonlocally coupled chaotic oscillators?," Europhys. Lett. 112, 40002 (2015).

${ }^{27}$ S. Ghosh, A. Kumar, A. Zakharova, and S. Jalan, "Birth and death of chimera: Interplay of delay and multiplexing," Europhys. Lett. 115, 60005 (2016).

${ }^{28}$ S. Bogomolov, G. Strelkova, E. Schöll, and V. Anishchenko, "Amplitude and phase chimeras in an ensemble of chaotic oscillators," Tech. Phys. Lett. 42, 765-768 (2016).

${ }^{29}$ T. E. Vadivasova, G. I. Strelkova, S. A. Bogomolov, and V. S. Anishchenko, "Correlation analysis of the coherence-incoherence transition in a ring of nonlocally coupled logistic maps," Chaos 26, 093108 (2016).

${ }^{30}$ S. A. Bogomolov, A. V. Slepnev, G. I. Strelkova, E. Schöll, and V. S. Anishchenko, "Mechanisms of appearance of amplitude and phase chimera states in ensembles of nonlocally coupled chaotic systems," Commun. Nonlinear Sci. Numer. Simul. 43, 25-36 (2017).

${ }^{31}$ I. A. Shepelev, A. V. Bukh, G. I. Strelkova, T. E. Vadivasova, and V. S. Anishchenko, "Chimera states in ensembles of bistable elements with regular and chaotic dynamics," Nonlinear Dyn. 90, 2317-2330 (2017).

${ }^{32} \mathrm{~N}$. Semenova, G. Strelkova, V. Anishchenko, and A. Zakharova, "Temporal intermittency and the lifetime of chimera states in ensembles of nonlocally coupled chaotic oscillators," Chaos 27, 061102 (2017).

${ }^{33}$ N. I. Semenova, E. V. Rybalova, G. I. Strelkova, and V. S. Anishchenko, "Coherence-incoherence transition in ensembles of nonlocally coupled chaotic oscillators with nonhyperbolic and hyperbolic attractors," Regul. Chaotic Dyn. 22, 148-162 (2017).

${ }^{34}$ A. Bukh, E. Rybalova, N. Semenova, G. Strelkova, and V. Anishchenko, "New type of chimera and mutual synchronization of spatiotemporal structures in two coupled ensembles of nonlocally interacting chaotic maps," Chaos 27, 111102 (2017).

${ }^{35}$ J. D. Hart, D. C. Schmadel, T. E. Murphy, and R. Roy, "Experiments with arbitrary networks in time-multiplexed delay systems," Chaos 27, 121103 (2017).

${ }^{36}$ E. V. Rybalova, G. I. Strelkova, and V. S. Anishchenko, "Mechanism of realizing a solitary state chimera in a ring of nonlocally coupled chaotic maps," Chaos Solitons Fractals 115, 300 (2018).

${ }^{37}$ S. Ghosh, A. Zakharova, and S. Jalan, "Non-identical multiplexing promotes chimera states," Chaos Solitons Fractals 106, 56-60 (2018).

${ }^{38}$ A. zur Bonsen, I. Omelchenko, A. Zakharova, and E. Schöll, "Chimera states in networks of logistic maps with hierarchical connectivities," Eur. Phys. J. B 91, 65 (2018).

${ }^{39}$ G. I. Strelkova, T. E. Vadivasova, and V. S. Anishchenko, "Synchronization of chimera states in a network of many unidirectionally coupled layers of discrete maps," Regul. Chaotic Dyn. 23, 948-960 (2018).

${ }^{40}$ I. A. Shepelev, A. Bukh, T. E. Vadivasova, V. S. Anishchenko, and A. Zakharova, "Double-well chimeras in 2D lattice of chaotic bistable elements," Commun. Nonlinear Sci. Numer. Simul. 54, 50-61 (2018).

${ }^{41}$ E. Rybalova, T. Vadivasova, G. Strelkova, V. Anishchenko, and A. Zakharova, "Forced synchronization of a multilayer heterogeneous network of chaotic maps in the chimera state mode," Chaos 29, 033134 (2019).

${ }^{42} \mathrm{P}$. Chandran, R. Gopal, V. Chandrasekar, and N. Athavan, "Chimera states in coupled logistic maps with additional weak nonlocal topology," Chaos 29, 053125 (2019).

${ }^{43}$ M. Winkler, J. Sawicki, I. Omelchenko, A. Zakharova, V. Anishchenko, and E. Schöll, "Relay synchronization in multiplex networks of discrete maps," Europhys. Lett. 126, 50004 (2019).

${ }^{44}$ Y. Zhang, Z. G. Nicolaou, J. D. Hart, R. Roy, and A. E. Motter, "Critical switching in globally attractive chimeras," arXiv:1911.07871 (2019).

${ }^{45}$ C. R. Nayak and N. Gupte, "Chimera states in coupled sine-circle map lattices," AIP Conf. Proc. 1339, 172-180 (2011).

${ }^{46} \mathrm{~J}$. Singha and N. Gupte, "Spatial splay states and splay chimera states in coupled map lattices,” Phys. Rev. E 94, 052204 (2016).

${ }^{47} \mathrm{~K}$. Kaneko, "Clustering, coding, switching, hierarchical ordering, and control in a network of chaotic elements," Physica D 41, 137 (1990).

${ }^{48} \mathrm{~K}$. Kaneko and I. Tsuda, "Chaotic itinerancy," Chaos 13, 926 (2003).

${ }^{49}$ H.-O. Peitgen, H. Jürgens, and D. Saupe, Chaos and Fractals: New Frontiers of Science (Springer Science \& Business Media, 2006).

${ }^{50} \mathrm{The} \sigma$ and $\delta$ measures are based on differences of values of the quadratic map, which are of the order $10^{\circ}$. Accordingly, values of these measures on the order of $10^{-14}-10^{-16}$ are just above the resolution of the double floating point precision. ${ }^{59}$ We, therefore, assume that such nonzero but very small values arise from the limited floating point precision and report them to be zero. Any value $\geq 10^{-13}$ is reported as nonzero with two significant digits.

${ }^{51}$ We specify the $C_{\mathrm{w}}$ and $C_{\mathrm{a}}$ values with two significant digits. Due to the chaotic nature of the dynamics, one needs the exact values and initial conditions at double floating point precision to reproduce our results. These will be provided along with the source codes to run our simulations on the institutional web site of the first author.

${ }^{52}$ E. A. Martens, M. J. Panaggio, and D. M. Abrams, "Basins of attraction for chimera states," New J. Phys. 18, 022002 (2016).

${ }^{53}$ S. Rakshit, B. K. Bera, M. Perc, and D. Ghosh, "Basin stability for chimera states," Sci. Rep. 7, 1-12 (2017).

${ }^{54}$ Z. Faghani, Z. Arab, F. Parastesh, S. Jafari, M. Perc, and M. Slavinec, "Effects of different initial conditions on the emergence of chimera states," Chaos Solitons Fractals 114, 306-311 (2018).

${ }^{55}$ Y. Maistrenko, B. Penkovsky, and M. Rosenblum, "Solitary state at the edge of synchrony in ensembles with attractive and repulsive interactions," Phys. Rev. E 89, 060901 (2014)

${ }^{56}$ O. E. Omel'chenko, M. Wolfrum, and Y. L. Maistrenko, "Chimera states as chaotic spatiotemporal patterns,” Phys. Rev. E 81, 065201 (2010).

${ }^{57} \mathrm{P}$. Liu, "Control and synchronization of Mandelbrot sets in coupled map lattice," Nonlinear Dyn. 73, 299-310 (2013).

${ }^{58} \mathrm{D}$. Wang, S. Liu, Y. Zhao, and C. Jiang, "Control of the spatial Mandelbrot set generated in coupled map lattice," Nonlinear Dyn. 84, 1795-1803 (2016).

${ }^{59}$ IEEE Standards Committee, "754-2008 IEEE standard for floating-point arithmetic," IEEE Comput. Soc. Std 2008, 1-70. 